\title{
Q-Learning-Driven BP decoding for Polar Codes
}

\author{
Lucas M. de Oliveira, Robert M. Oliveira and Rodrigo C. de Lamare
}

\begin{abstract}
This paper presents an enhanced belief propagation (BP) decoding algorithm and a reinforcement learning-based BP decoding algorithm for polar codes. The enhanced BP algorithm weighs each Processing Element (PE) input based on their signals and Euclidean distances using a heuristic metric. The proposed reinforcement learning-based BP decoding strategy relies on reweighting the messages and consists of two steps: we first weight each PE input based on their signals and Euclidean distances using a heuristic metric, then a Q-learning algorithm (QLBP) is employed to figure out the best correction factor for successful decoding. Simulations show that the proposed enhanced BP and QLBP decoders outperform the successive cancellation (SC) and belief propagation (BP) decoders, and approach the SCL decoders.
\end{abstract}

Keywords-Q-Learning, Reinforcement Learning, Belief Propagation, Polar Codes.

\section{INTRODUTION}

Polar codes, originally introduced in 2009 by Arikan [1], are a significant breakthrough in coding theory. They are theoretically proven capacity-achieving codes based on the general channel polarization phenomenon [1]. As part of the 5G New Radio enhanced mobile broadband (eMBB) standard, significant research efforts have been made to design satisfactory decoders to meet low-latency and high-speed requirements, ranging from efficient decoding to suitable hardware implementation.

One of the first decoders that arose was the Successive Cancellation (SC) decoder [1], which can achieve good errorcorrecting capability with low complexity. However, this decoder often exhibits low performance due to the type of SC-based decoding that is characterized by serial message updating, propagation errors and low capacity for high-speed real-time applications. Therefore, the successive cancellation list (SCL) decoding [2] was proposed to improve the errorcorrection performance of SC, since it stores the most likely codewords in a list, reducing error probability and improving the performance. Moreover, SCL can be further enhanced by concatenating a cyclic redundancy check (CRC) code [2]. As can be seen in [3] - [5], CRC-aided successive cancellation list (CA-SCL) decoding attains promising error-correction performance.

Furthermore, several attempts have been made to reduce the computational complexity and increase the throughput of SC and SCL decoders. Inherited from Low-Density ParityCheck (LDPC) codes, Belief Propagation (BP) decoders were introduced in [6], because of their particular advantages with

Lucas M. de Oliveira, Robert M. Oliveira and Rodrigo C. de Lamare - Centre for Telecommunications Studies (CETUC), Pontifical Catholic University of Rio de Janeiro (PUC-Rio), Rio de Janeiro-RJ, Brasil, E-mails: lucasmarques@cetuc.puc-rio.br, rbtmota@gmail.com and delamare@cetuc.puc-rio.br. This work was supported by CNPq, CAPES, FAPERJ and FAPESP. respect to parallelism, high throughput, and low latency. Nevertheless, due to their characteristics, BP decoding requires a large number of iterations to achieve good performance. Thus, a way to improve the performance is to employ BP list decoding [7], which operates when the standard polar code factor graph fails to produce the correct decoding result and the permuted version of the standard graph may yield the correct estimate.

In this paper, we propose an enhanced BP algorithm and a Q-Learning BP (QLBP) approach to enhance BP decoding of polar codes. Initially, a weighting technique based on the Euclidean distance and the signal of the Processing Element inputs is presented and incorporated into a BP strategy to devise the enhanced BP algorithm. Then, based on the fact that a correction factor can enhance the weighting process, the QLBP is devised to compute the best factor and to ensure an optimized decoding performance. Numerical results show that the proposed QLBP algorithm outperforms the proposed enhanced $\mathrm{BP}$, the existing $\mathrm{BP}$ and the SC decoding algorithms.

The remainder of this paper is organized as follows. Section II introduces polar codes and the decoding problem with BP. Section III presents the Enhanced BP algorithm and its weighting method. Section IV proposes a Q-learning strategy for computing BP weights, then presents the Q-Learning driven BP decoding algorithm. Section V presents the simulated results. Conclusions are drawn in Section VI.

\section{PRELiminaries}

\section{A. Polar Codes}

Polar codes are derived from channel combination and polarization theory. As the code length $\mathrm{N}=2^{n}$ gets larger through splitting and combining channels, the symmetric capacity of bit-channels tends to either 1 or 0 . In that way, there are basically two types of channels: noiseless channels, closer to the capacity of the binary symmetric channels and denoted by the set $\mathcal{A}$, and noisy channels, denoted by the set $\mathcal{A}^{\mathrm{c}}$. Let $\mathbf{u}_{1}^{n}=\left\{u_{1}, u_{2}, \ldots, u_{n}\right\}$ denote the source vector and $\mathbf{x}_{1}^{n}=\left\{x_{1}, x_{2}, \ldots, x_{n}\right\}$ denote the code word vector. For polar codes with $\mathrm{PC}(\mathrm{N}, \mathrm{K}), \mathrm{R}=\frac{\mathrm{K}}{\mathrm{N}}$, the vector $\mathbf{u}$ consists of $\mathrm{K}$ information bits in $\mathcal{A}$ and $\mathrm{N}-\mathrm{K}$ frozen bits in $\mathcal{A}^{\mathrm{c}}$. The encoding process of polar codes, defined by Arikan, can be expressed by $\mathbf{x}_{1}^{n}=\mathbf{u}_{1}^{n} \mathbf{G}^{\otimes n}$, where $\mathbf{G}=\left(\begin{array}{ll}1 & 0 \\ 1 & 1\end{array}\right)$ is the n-th Kronecker power of the polarizing matrix $\mathbf{G}$ and $\mathbf{n}=\log _{2} \mathrm{~N}$.

\section{B. Belief Propagation Decoding}

The BP decoder is a message-passing decoder with iterative processing over the factor graph of any polar code $\mathrm{PC}(\mathrm{N}, \mathrm{K})$. The factor graph is based on corresponding polarization matrix $\mathbf{G}^{\otimes n}$, composed of $n=\log _{2} N$ stages, each one with $N / 2$ 
processing elements (PEs), and $(n+1) N$ nodes. Two types of LLRs are transmitted over the factor graph: the left-to-right message $R_{i, j}^{(t)}$ and the right-to-left message $L_{i, j}^{(t)}$, where $i, j$ denotes the $\mathrm{j}$-th node at the $\mathrm{i}$-th stage whereas $t$ denotes the t-th iteration.

Considering a binary phase-shift keying (BPSK) modulation and an additive white Gaussian noise (AWGN) channel model, the noisy received code word is given by

$$
\mathbf{y}=(\mathbf{1}-2 \mathbf{x})+\mathbf{z}
$$

where $\mathbf{1}$ is an all-one vector, $\mathbf{z}$ is the AWGN noise vector with variance $\sigma^{2}$ and zero mean. In LLR domain, the LLR inputs for BP decoding of polar codes are initialized as:

$$
\begin{aligned}
& L_{n+1, j}^{(0)}=\ln \frac{P_{r}\left(x_{j}=0 \mid y_{j}\right)}{P_{r}\left(x_{j}=1 \mid y_{j}\right)}=\frac{2 y_{j}}{\sigma^{2}} \\
& R_{1, j}^{(0)}= \begin{cases}0 & \text { if } \mathrm{j} \in \mathbb{A} \\
\infty & \text { if } \mathrm{j} \in \overline{\mathbb{A}}\end{cases}
\end{aligned}
$$

where $x_{j}$ and $y_{j}$ denote the $\mathrm{j}$-th bit of modulated and received codeword, respectively.

The forward and backward propagation of the LLRs over the PEs, shown in Fig. II-B, is based on the following iterative updating rules:

$$
\begin{aligned}
& L_{i, j}^{(t)}=g\left(L_{i+1, j}^{(t-1)}, L_{i+1, j+N / 2^{i}}^{(t-1)}+R_{i, j+N / 2^{i}}^{(t-1)}\right) \\
& L_{i, j+N / 2^{i}}^{(t)}=g\left(L_{i+1, j}^{(t-1)}, R_{i, j}^{(t-1)}\right)+L_{i+1, j+N / 2^{i}}^{(t-1)} \\
& R_{i+1, j}^{(t)}=g\left(R_{i, j}^{(t-1)}, L_{i+1, j+N / 2^{i}}^{(t-1)}+R_{i, j+N / 2^{i}}^{(t-1)}\right) \\
& R_{i+1, j+N / 2^{i}}^{(t)}=g\left(L_{i+1, j}^{(t-1)}+R_{i, j}^{(t-1)}\right)+R_{i, j+N / 2^{i}}^{(t-1)}
\end{aligned}
$$

where $g(x, y)$ is referred to as the operator:

$$
\begin{aligned}
g(x, y) & =\ln \frac{1+e^{x+y}}{e^{x}+e^{y}} \\
& \approx 0.9375 \cdot \operatorname{sign}(x) \cdot \operatorname{sign}(y) \cdot \min (|x|,|y|)
\end{aligned}
$$

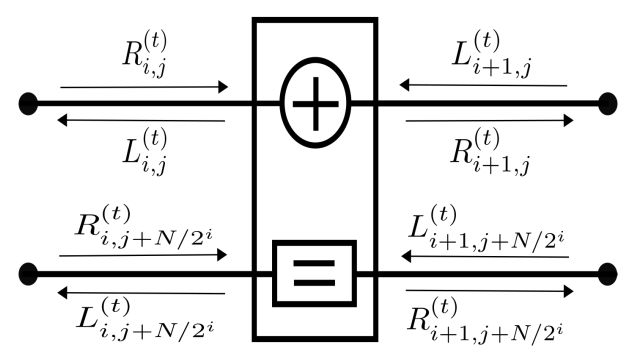

Fig. 1. Processing element update

When the maximum number of iterations, $T_{\max }$, is reached, the information bit $\hat{\mathbf{u}}_{j}$ and the transmitted codeword $\hat{\mathbf{x}}_{j}$ are estimated based on their LLRs using the following hard decision criteria:

$$
\begin{aligned}
& \hat{u}_{j}= \begin{cases}0, & \text { if } L_{1, j}^{T_{\max }}+R_{1, j}^{T_{\max }}>0 \\
1, & \text { otherwise }\end{cases} \\
& \hat{x}_{j}= \begin{cases}0, & \text { if } L_{n+1, j}^{T_{\max }}+R_{n+1, j}^{T_{\max }}>0 \\
1, & \text { otherwise }\end{cases}
\end{aligned}
$$

\section{ENHANCED BP DECODING}

In this section, we propose a weighting technique for $\mathrm{BP}$ decoders. This technique will lay the foundation for the Qlearning algorithm, which will be discussed later. As can be seen in Eq. (3), the propagation of the messages requires four parameters for each direction, which is, $L_{i+1, j}^{(t)}, L_{i+1, j+N / 2^{i}}^{(t)}$ $R_{i, j}^{(t)}$ and $R_{i, j+N / 2^{i}}^{(t)}$ for $\mathrm{L}$ messages and $R_{i, j}^{(t)}, R_{i, j+N / 2^{i}}^{(t)}$ $L_{i+1, j}^{(t)}$ and $L_{i+1, j+N / 2^{i}}^{(t)}$ for R messages. Moreover, as the PEs updates can be summarized in signal and modules successive operations defined by Eq. (3), our proposed weighting technique is built on how those four LLRs evolve in terms of signal and module over time.

Thus, we introduce four weighting factors, $\rho_{1}, \rho_{2}, \rho_{3}$, and $\rho_{4}$, which will modify how the LLRs are updated, as can be seen below.

$$
\begin{aligned}
& L_{i, j}^{(t)}=g\left(\rho_{1} \cdot L_{i+1, j}^{(t-1)}, \rho_{1}\left(L_{i+1, j+N / 2^{i}}^{(t-1)}+R_{i, j+N / 2^{i}}^{(t)}\right)\right) \\
& L_{i, j+N / 2^{i}}^{(t)}=g\left(\rho_{2} \cdot L_{i+1, j}^{(t-1)}, \rho_{2} \cdot R_{i, j}^{(t)}\right)+\rho_{2} \cdot L_{i+1, j+N / 2^{i}}^{(t-1)}
\end{aligned}
$$

where

$$
\begin{aligned}
& \rho_{1}=1+\beta \cdot\left[\frac{|| L_{i, j}^{(t)}|-| L_{i, j}^{(t-1)}||}{\left(\left|L_{i, j}^{(t)}\right|+\left|L_{i, j}^{(t-1)}\right|\right)}\right] \cdot \Delta_{1} \\
& \rho_{2}=1+\beta \cdot\left[\frac{|| L_{i, j+N / 2^{i}}^{(t)}|-| L_{i, j+N / 2^{i}}^{(t-1)} \mid}{\left(\left|L_{i, j+N / 2^{i}}^{(t)}\right|+\left|L_{i, j+N / 2^{i}}^{(t-1)}\right|\right)}\right] \cdot \Delta_{2} \\
& \Delta_{1}=\operatorname{sign}\left(L_{i, j}^{(t)}+L_{i, j}^{(t-1)}\right) \\
& \Delta_{2}=\operatorname{sign}\left(L_{i, j+N / 2^{i}}^{(t)}+L_{i, j+N / 2^{i}}^{(t-1)}\right)
\end{aligned}
$$

The weighting method is based on the distance between the LLRs at the time $t$ and $t-1$ and whether their signals have changed over the iterations. Note that when $\left|L_{i, j}^{(t)}\right|$ and $\left|L_{i, j}^{(t-1)}\right|$ are close to each other, $\rho_{1}$ is approximately equal to 1. Consequently, the node update is similar to the Eq. 3. Thus, as these values deviate, the greater the weighting. Moreover, the signal deviations are considered by $\Delta_{1}$. It should also be pointed out that the same idea is applied to the subsequent weighting factors.

$$
\begin{aligned}
& R_{i+1, j}^{(t)}=g\left(\rho_{3} \cdot R_{i, j}^{(t)}, \rho_{3}\left(L_{i+1, j+N / 2^{i}}^{(t-1)}+R_{i, j+N / 2^{i}}^{(t)}\right)\right) \\
& R_{i+1, j+N / 2^{i}}^{(t)}=g\left(\rho_{4}\left(L_{i+1, j}^{(t-1)}+R_{i, j}^{(t)}\right)\right)+\rho_{4} \cdot R_{i, j+N / 2^{i}}^{(t)}
\end{aligned}
$$

where

$$
\begin{aligned}
& \rho_{3}=1+\beta \cdot\left[\frac{|| R_{i+1, j}^{(t)}|-| R_{i+1, j}^{(t-1)}||}{\left(\left|R_{i+1, j}^{(t)}\right|+\left|R_{i+1, j}^{(t-1)}\right|\right)}\right] \cdot \Delta_{3} \\
& \rho_{4}=1+\beta \cdot\left[\frac{|| R_{i+1, j+N / 2^{i}}^{(t)}|-| R_{i+1, j+N / 2^{i}}^{(t-1)} \mid}{\left(\left|R_{i+1, j+N / 2^{i}}^{(t)}\right|+\left|R_{i+1, j+N / 2^{i}}^{(t-1)}\right|\right)}\right] \cdot \Delta_{4} \\
& \Delta_{3}=\operatorname{sign}\left(R_{i+1, j}^{(t)}+R_{i+1, j}^{(t-1)}\right) \\
& \Delta_{4}=\operatorname{sign}\left(R_{i+1, j+N / 2^{i}}^{(t)}+R_{i+1, j+N / 2^{i}}^{(t-1)}\right)
\end{aligned}
$$


Furthermore, it is worth noting that $\beta$ is a general correction factor for all processing elements, whose simulations have shown that it must belong to the range $[-0.50,0.50]$. Thus, an open problem is how to set up the best $\beta$ for a specific input, a task in which the Q-learning algorithm, proposed in the next section, tries to solve.

A high-level description of the enhanced BP decoding algorithm is illustrated in Algorithm 1. The algorithm takes the received codeword $\mathrm{y}_{1}^{n}$, the code block length $\mathrm{N}$, the maximum number of iterations $T_{\max }$, and the information set $\mathcal{A}$ and calculates the estimated free bits $\hat{\mathbf{u}}_{\mathcal{A}}$ as an output vector.

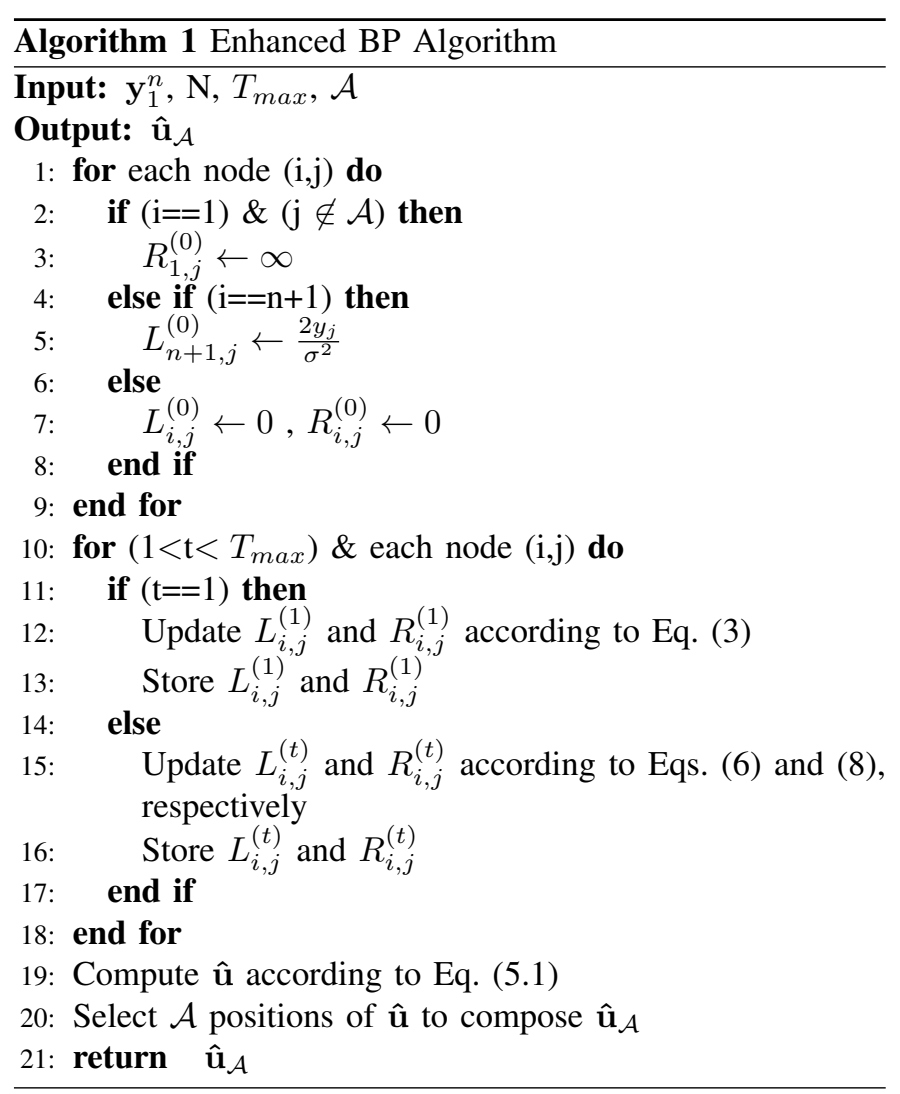

\section{Proposed Q-Learning BP Decoding}

\section{A. Reinforcement learning and Q-Learning}

Reinforcement Learning, RL, is an area of machine learning in which an agent learns how to take actions from its action space, within a particular environment, in order to maximize rewards over time. At each time step, the agent, which is undergoing the learning process, is in a state $s_{t}$, selects an action $a_{t}$ and moves to the next state $s_{t+1}$, while obtaining a reward $r_{t}$. The aim of learning is to train the agent to find an optimal policy, which is a mapping between states and actions, and will return the maximum cumulative rewards from taking a series of actions in one or more states.

A Markov Decision Process [8], MDP, is a mathematical framework for fully observable sequential decision making problems in stochastic environments. Defined as a 5-tuple, $\left(S, A, R, P\left(s, a, s^{\prime}\right), R\left(s, a, s^{\prime}\right)\right), S$ represents a set of states, where $s_{t} \in S$ is the state at time-step $t, A$ is a finite set of actions, where $a_{t} \in A$ is the action executed at time-step $t, P\left(s, a, s^{\prime}\right)$ is the probability that action $a$ in state $s$ at time $t$ will lead to state $s^{\prime}$ at time $t+1$, and $R\left(s, a, s^{\prime}\right)$ is the immediate reward received after a transition from state $s$ to $s^{\prime}$ due to action $a$.

Q-Learning [9], a model-free reinforcement learning algorithm, is used to learning the optimal policy of an agent without using or estimating the dynamics of the environment. For every state-action pair, a $\mathrm{Q}$-value, $\mathrm{Q}(s, a)$, measures the total amount of discounted rewards expected over the future when the agent moves from the state $s_{t}$ to the state $s_{t+1}$ with $a_{t}$ and sticks to its policy afterwards. The Q-learning update rule assumes the following general form:

$$
\begin{aligned}
Q^{n}\left(s_{t}, a_{t}\right) & =Q^{o}\left(s_{t}, a_{t}\right)+\alpha \delta \\
\delta & =\left[r_{t}+\gamma \cdot \max _{a} Q\left(s_{t+1}, a\right)-Q\left(s_{t}, a_{t}\right)\right]
\end{aligned}
$$

where $\alpha$ is the learning rate, and affects how the Q-values are altered after taking an action. The constant $\gamma$ is the discount factor, and determines how much influence the future rewards have on the updates of the Q-values. $\alpha=0.1$ and $\gamma=0.6$ were chosen because they presented better results.

\section{B. Q-Learning BP Decoding}

As mentioned in Section III, an open problem is how to compute the best value of $\beta$. However, instead of having a general value of $\beta$ to all PEs on the factor graph, we evaluated different values of $\beta$ for each PE in order to avoid a huge state subspace. From this approach, our environment is composed by each $\mathrm{PE}_{l, m}, l, m$ denotes $\mathrm{m}$-th $\mathrm{PE}$ at the 1-stage, where $m$ belongs to the range $\left[1, \frac{N}{2}\right]$ and $l$ belongs to the range $[1, n]$. Thus, the agent, in a given state $s_{t_{l, m}}$, selects an action $a_{t_{l, m}}$ and waits for the decoding process to return to $\mathrm{PE}_{l, m}$ to be ultimately rewarded.

\section{- Reward:}

The reward quantifies the desirability of choosing an action while transitioning to some state. It can be either positive or negative, the latter being interpreted as a penalty for an undesirable action. The total reward with the discount factor that the agent will achieve from the current time step t to the end of the task can be defined as:

$$
R_{t}=r_{t}+\gamma r_{t+1}+\ldots+\gamma^{n-t} r_{n}=r_{t}+\gamma R_{t+1}
$$

We have implemented a high positive reward for a successful decoding process in order to encourage the agent to achieve this goal. In addition, the agent should obtain a slight positive reward if the LLR at time $t$ has the same signal of the LLR at time $t-1$ in a given PE. On the other hand, if the LLR at time $t$ and $t-1$ has not the same signal, the agent should obtain a slight negative reward. In doing so, we avoid undesirable actions, which keep away from successful decoding. The discount factor and the rewards values are shown in Table I.

Note that when we have successful decoding, the reward can be either 20,10, or 0. As can be seen in Section II-B, the propagation of the LLRs over the PEs involves four numbers and generates two output LLRs for each propagation direction. 
TABLE I

\begin{tabular}{cc}
\hline \hline Event & Reward \\
\hline Successful decoding & $20 / 10 / 0$ \\
LLRs have the same signal & 1 \\
LLRs have not the same signal & -1 \\
\hline
\end{tabular}

Therefore, if both output LLRs have not changed their signal over time, we reward the associated PE with 20. If one of them has changed its signal over time, we reward the associated PE with 10. On the other hand, if both output LLRs have changed their signal, we reward with 0 . Any other reward values could be chosen, but they should have the same logic.

\section{- State Space:}

The state space is the set of all possible situations a processing element could have. For each propagation, it is necessary four numbers to compute the outputs. Thus, there are $2^{4}$ signal variations and 4 ! modules variations, which means a state space with $4 ! \cdot 2^{4}=384$ possible states.

\section{- Actions:}

Note that the agent cannot control so far what state it ends up in, since it can be influenced by choosing some action $a$. Thus, focusing on a single state $s$ and action $a$, we introduce recursively the $\mathrm{Q}$ function, $Q(s, a)$, in terms of the Q-value of the next state $s^{\prime}$, which can be expressed as follows:

$$
Q(s, a)=r+\gamma \cdot \max _{a^{\prime}} Q\left(s^{\prime}, a^{\prime}\right)
$$

Also known as the Bellman equation, Eq. (12) tells us that the maximum future reward is given by the reward that the agent received for entering the current state $s$ plus the maximum future reward for the next state $s^{\prime}$.

In the proposed QLBP algorithm, during the decoding process, for each $\mathrm{PE}$ in the factor graph, the agent encounters one of the 384 states and it takes an action. The action in our case can be a value between -0.5 and 0.5 . If the signal of the LLR at time $t$ is different from the LLR at $t-1$, the choice of an action will change how the LLR is weighted according to Eqs. (7) and (9). Otherwise, it is assumed $\rho_{1,2,3,4}=1$.

An agent could interact with the environment in 2 different ways. The first one is to use a lookup table with state-action pairs to store and get information. Each state-action pair is associated with a Q-value that indicates the quality of the decision. Thus, in a given state $s^{*}$, the agent selects the action based on the maximum value of $\mathrm{Q}\left(s^{*}, a\right)$. This procedure is known as exploiting since the information that we have available is used to make a decision.

The second way to take action is to act randomly. This is called exploring and the exploration method used is the greedy approach [10]. Instead of selecting actions based on the maximum future reward, we select an action at random. Acting randomly is important because it allows the agent to explore and discover new states that otherwise may not be selected during the exploitation process. It is possible to balance exploration and exploitation using $\epsilon$, which measures how often you want to explore instead of exploit. It was used $\epsilon=0.5$.

A high-level description of the QLBP algorithm is depicted in Algorithm 2. The algorithm takes the conventional BP parameters (such as the received codeword $\mathbf{y}_{1}^{n}$, the code block length $\mathrm{N}$, the maximum number of iterations $T_{\max }$, and the information set $\mathcal{A}$ ), the Q-Table and the Q-Learning parameters (such as learning rate $\alpha$, discount factor $\gamma$, and $\epsilon$ greedy parameter), and outputs the estimated free bits $\hat{\mathbf{u}}_{\mathcal{A}}$ and the actualized Q-Table.

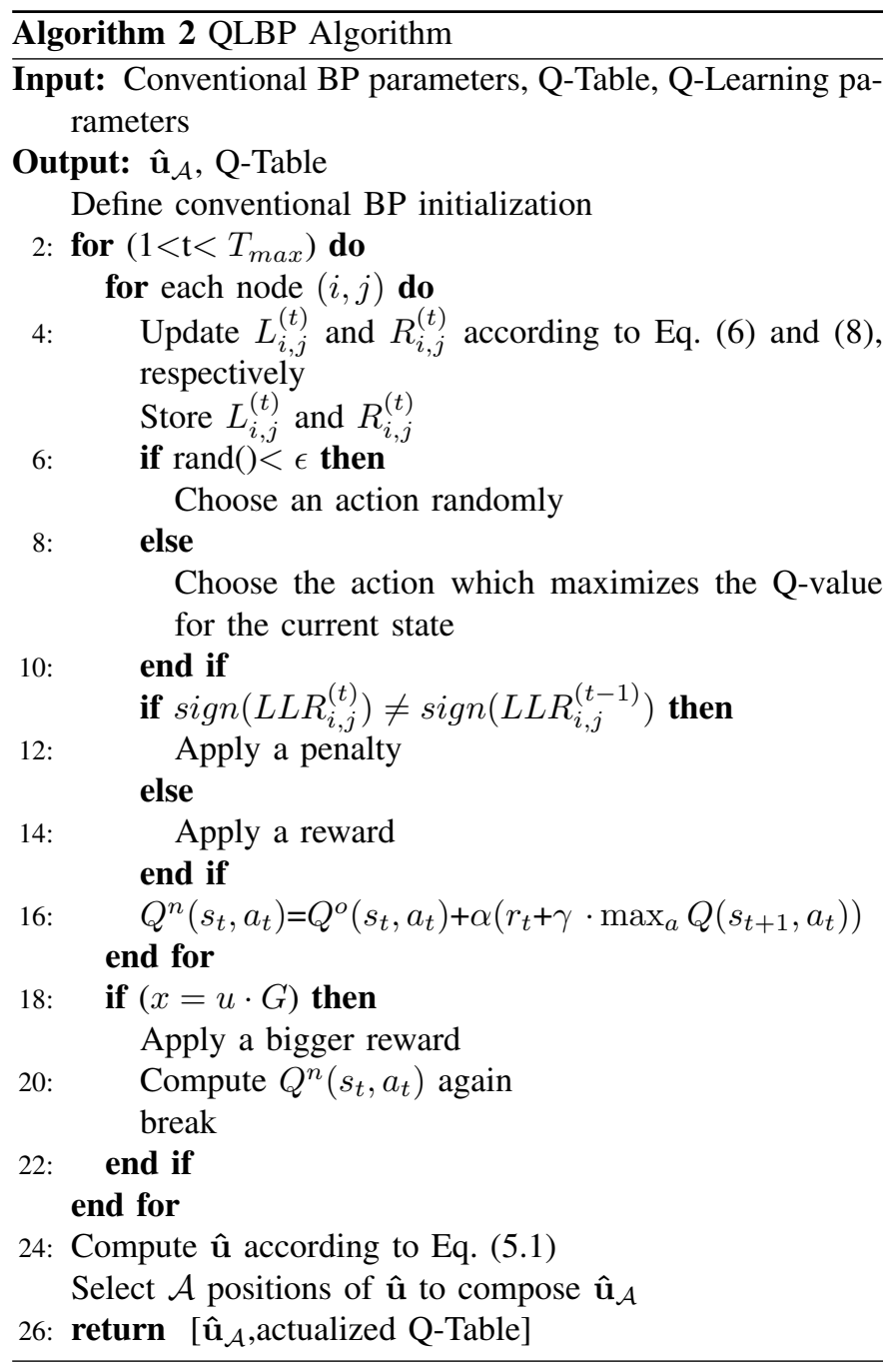

\section{Simulations}

In this section, the simulation results are presented to demonstrate the effectiveness of the proposed enhanced BP and QLBP algorithms compared to different decoders, namely Arikan's original SC, SCL with List-4 and List-8 and Arikan's original $\mathrm{BP}$ for $\mathrm{N}=256, \mathrm{~N}=512$ and $\mathrm{R}=\frac{1}{2}$.

As can be seen in Figs. 2 and 3, for $\mathrm{N}=256, \mathrm{R}=\frac{1}{2}$, both results have shown that the proposed Q-Learning-Driven BP decoder outperforms the SC, BP, and Enhanced BP algorithms by up to approximately $0.5 \mathrm{~dB}$ and $0.4 \mathrm{~dB}$ in terms of BER and FER, respectively, at $2 \mathrm{~dB}$, and approaches the performance of the benchmark decoder in the literature, SCL. However, note that the performance gain decreases as the signal-to-noise ratio increases. Thus, the proposed QLBP algorithm is more suitable to be implemented at lower SNRs at this code length. 


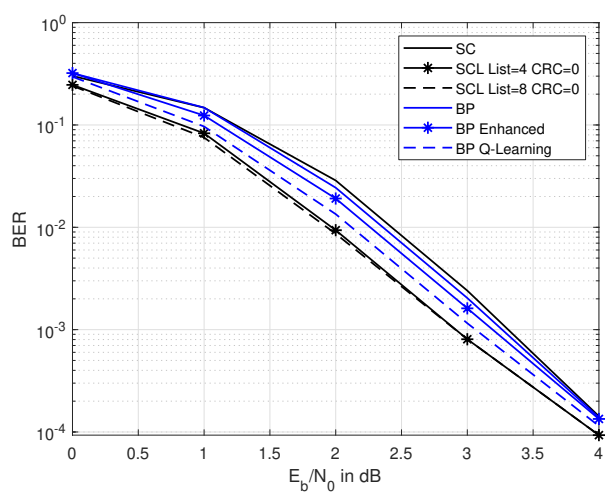

Fig. 2. BER comparison between Arikan's original SC, SCL with List-4 and List-8, Arikan's original BP, our proposed BP Enhanced and BP Q-learning decoders for $\mathrm{N}=256$ and $\mathrm{K}=128$; no $\mathrm{CRC}$ used.

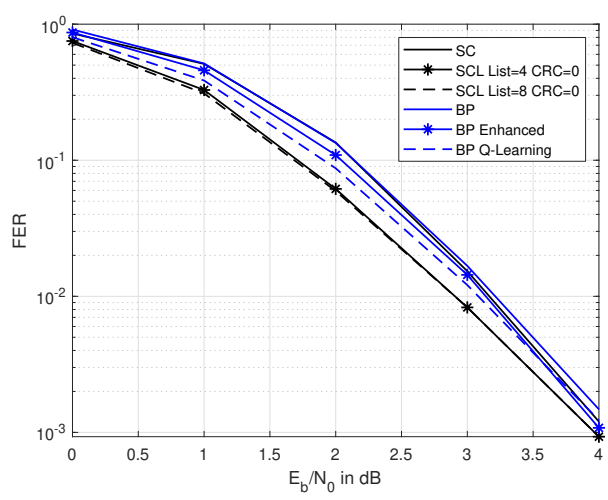

Fig. 3. FER comparison between Arikan's original SC, SCL with List-4 and List-8, Arikan's original BP, our proposed BP Enhanced and BP Q-learning decoders for $\mathrm{N}=256$ and $\mathrm{K}=128$; no $\mathrm{CRC}$ used.

In the second example, we assess the decoders for $\mathrm{N}=512$ and $\mathrm{K}=256$ in Figs. 4 and 5. As mentioned before, the proposed QLBP decoder also outperforms the SC, BP, and Enhanced BP algorithms by up to approximately $0.4 \mathrm{~dB}$ and $0.25 \mathrm{~dB}$ in terms of BER and FER, respectively, at $2 \mathrm{~dB}$. Besides that, although the performance of the SCL decoder has not been achieved, the proposed QLBP decoder performance has got even closer to the benchmark decoder and renders itself more easily to implementation. The performance gain of BER still maintained constant over the SNRs even though the FER has not.

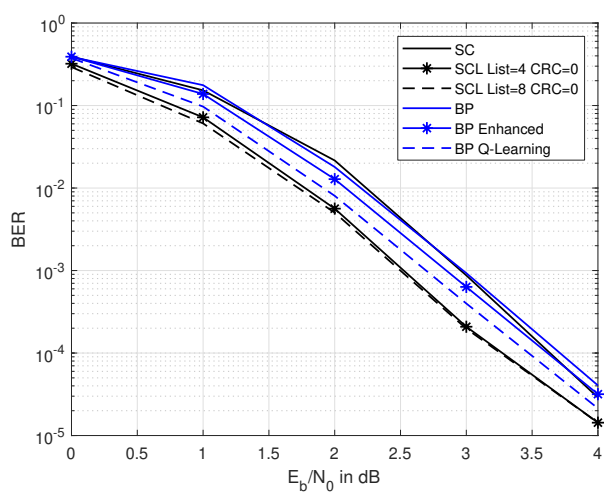

Fig. 4. BER comparison between Arikan's original SC, SCL with List-4 and List-8, Arikan's original BP, our proposed BP Enhanced and BP Q-learning decoders for $\mathrm{N}=512$ and $\mathrm{K}=256$; no $\mathrm{CRC}$ used.

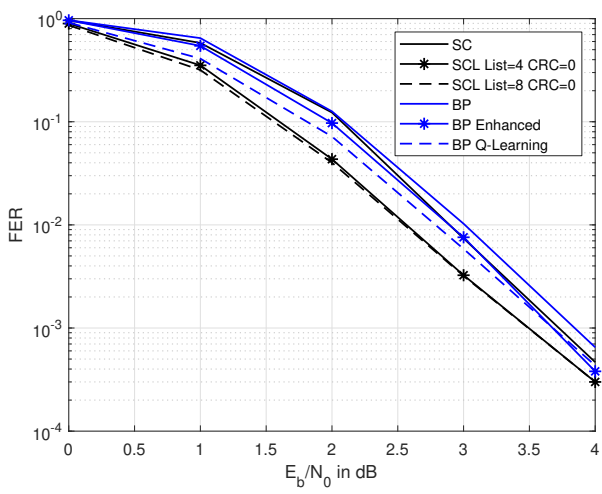

Fig. 5. FER comparison between Arikan's original SC, SCL with List-4 and List-8, Arikan's original BP, our proposed BP Enhanced and BP Q-learning decoders for $\mathrm{N}=512$ and $\mathrm{K}=256$; no $\mathrm{CRC}$ used.

Future works will consider puncturing techniques [11], multiple-antenna systems [12], [13].

\section{CONCLUSION}

This paper exploits the design of a BP decoder driven by Q-Learning, which seeks the best action, a weighting factor, for a specific state, an input in the processing element. More specifically, from our experience, Q-learning learns the optimal policy that maximizes the total reward, that is, a successful decoding. Thus, in the long term, the decoder learns how to weigh each processing element. Finally, simulations have shown that the performance of the proposed QLBP decoder for Polar Codes is better than Arikan's SC and BP codes, and approaches significantly the SCL decoders.

\section{REFERENCES}

[1] E. Arikan, "Channel Polarization: A Method for Constructing CapacityAchieving Codes for Symmetric Binary-Input Memoryless Channels", IEEE Transactions on Information Theory, vol. 55 no. 7, pp. 3051-3073, July 2009.

[2] I. Tal and A. Vardy, "List Decoding of Polar Codes", IEEE Transactions on Information Theory, vol. 61, no. 5, pp. 2213-2226, May 2015.

[3] A. Balatsoukas-Stimming, M. Bastani Parizi, and A. Burg, "LLR-based successive cancellation list decoding of polar codes," IEEE Trans. Signal Process., vol. 63, no. 19, pp. 5165-5179, Oct. 2015.

[4] J. Lin, C. Xiong, and Z. Yan, "A high throughput list decoder architecture for polar codes," IEEE Trans. Very Large Scale Integr. (VLSI) Syst., vol 24, no. 6, pp. 2378-2391, Jun. 2016

[5] D. Kim and I.-C. Park, "A fast successive cancellation list decoder for polar codes with an early stopping criterion," IEEE Trans. Signal Process., vol. 66, no. 18, pp. 4971-4979, Sep. 2018.

[6] E. Arıkan, "Polar codes: A pipelined implementation," in Proc. Int Symp. Broad. Commun. (ISBC), 2010, pp. 11-14.

[7] A. Elkelesh, M. Ebada, S. Cammerer, and S. T. Brink, "Belief propagation decoding of polar codes on permuted factor graphs," in Proc. IEEE Wireless Commun. Netw. Conf. (WCNC), 2018, pp. 1-6

[8] Bauerle N, Rieder U. Markov Decision Processes. Jahresber Dtsch Math Ver. 2010; 112:217

[9] Watkins, C.J.C.H., Dayan, P. Q-learning. Mach Learn 8, 279-292 (1992)

[10] J. Groot Kormelink, M. Drugan, and M. Wiering, "Exploration methods for connectionist Q-learning in Bomberman," in Proceedings of the 10th International Conference on Agents and Artificial Intelligence, ICAART 2018, Volume 2, Funchal, Madeira, Portugal, 2018, pp. 355-362

[11] R. M. Oliveira and R. C. de Lamare, "Puncturing Based on Polarization for Polar Codes in 5G Networks", 2018 15th International Symposium on Wireless Communication Systems, Aug. 2018.

[12] R. C. de Lamare, "Massive MIMO Systems: Signal Processing Chal lenges and Future Trends", Radio Science Bulletin, December 2013

[13] W. Zhang, H. Ren, C. Pan, M. Chen, R. C. de Lamare, B. Du and J. Dai, "Large-Scale Antenna Systems With UL/DL Hardware Mismatch: Achievable Rates Analysis and Calibration", IEEE Trans. Commun., vol.63, no.4, pp. 1216-1229, April 2015. 